\title{
The Importance of a Multidisciplinary Approach to VAP Prevention: The Role of the Respiratory Therapist
}

Ventilator associated pneumonia (VAP) is one of the most common hospital acquired infection among patients in the ICU, with an estimated incidence of $10-$ $20 \%$ in some institutions. ${ }^{1}$ Despite extensive research, the implementation of VAP prevention measures or "bundles" in acute care hospitals, strategies of active surveillance and early detection, advances in antimicrobial therapy and stewardship, and national tracking methods (National Healthcare Safety Network), VAP continues to be substantially associated with patient morbidity and mortality. ${ }^{2}$ Although the attributable mortality of VAP remains unknown, it has the highest associated mortality rate (25-50\%) of all the hospital acquired infections. Despite declines in VAP rates nationally (National Healthcare Safety Network), multiple negative outcomes are associated with VAP, such as prolonged mechanical ventilation, longer hospital stays, excess use of antibiotics, increased utilization of healthcare resources, and excess healthcare costs. ${ }^{1-2}$ Since VAP is associated with worse outcomes, it remains a major focus of quality initiatives and patient safety efforts in the healthcare setting.

Most healthcare facilities implement a VAP "bundle" for prevention, with the most common elements being elevation of the head of the bed, oral care, daily interruption of sedation, evaluation for a spontaneous breathing trial, gastrointestinal prophylaxis, and thrombotic disease prevention; but with these bundle components, full adherence to all the elements is a major hurdle. ${ }^{3}$ Educational campaigns and alerts via electronic medical records can increase adherence. ${ }^{4}$ Reporting of adherence and VAP rates to providers, with comparison to colleagues, can also improve implementation of all the elements. ${ }^{3-4}$ Throughout these studies, the burden of adherence largely fell on nursing staff. Even the implementation of a spontaneous breathing trial was directed by the nursing staff and carried out by the respiratory therapist (RT). With advanced nursing care needs in the ICU, bundle adherence can suffer as the nursing staff is spread thin and implementation of the elements can be delayed.

In this issue of Respiratory CARe, Arroliga et al propose that a multidisciplinary team approach is paramount to ensure adherence to evidenced-based practices of a VAP bundle. ${ }^{5}$ In turn, a reduction in the incidence of VAP is reported. Rather than report on the bundle component adherence or the method of alerting and reporting adherence, Arroliga et al focused on how these components were implemented, with a specific focus on oral care. In a retrospective cohort study conducted in a single-center setting, VAP bundle adherence was evaluated with RT involvement. Historically, the oral care component of the bundle had been done by nurses, with hydrogen peroxide solution every 4 hours. However, poor adherence to oral care was observed (33\%), and the responsibility was transferred to the RTs. Around the same time of this shift in responsibility, they also switched to using chlorhexidine $2 \%$ solution every 12 hours.

See the Original Study on Page 688

Arroliga et al observed that when the RTs assumed the responsibility of oral care, adherence drastically improved, to above $97 \%$. Not only did adherence increase, but VAP declined as well (4.27/1,000 ventilator days vs 1.20/1,000 ventilator days). Declines in antibiotic days were also noted. Although this study could not definitively establish causality in the reduction of VAP rates, the RTs' assumption of oral care and the increased bundle adherence (along with a chlorhexidine solution change) achieved a moderate decline in VAP rates. The individual impact of the increased adherence under RT care, or of the change to chlorhexidine from hydrogen peroxide, is unknown; but the impact on VAP rates was substantial and highlights the importance of a multidisciplinary role for prevention.

Arroliga et al emphasize the importance of a multidisciplinary task force to deliver best practices, with responsibility shared between nursing and RTs. This study highlights the role RTs can play in VAP prevention outside of ventilator management and spontaneous breathing trial initiation. Their work can expand to the bedside team and directly impact VAP rates. In this center, RT involvement worked well, but in some institutions another team may be better suited for the role of oral care. The role of a single center study can be limiting, but in this case it highlights the importance of a multidisciplinary approach-working as a team toward 


\section{The Importance of a Multidisciplinary Approach to VAP PreVention}

a common goal to improve patient outcomes. Their message resonates with what Helen Keller once said: "Alone we can do so little; together we can do so much."

\section{Christian Sandrock MD MPH} Joan Daly RN

Division of Pulmonary and Critical Care University of California Davis School of Medicine Sacramento, California

Christian Sandrock MD MPH, Division of Pulmonary and Critical Care, University of California Davis School of Medicine, 4150 V Street, \#3400, Sacramento CA 95817. E-mail: cesandrock@ucdavis.edu.

The authors have disclosed no conflicts of interest.

DOI: $10.4187 /$ respcare. 01855

\section{REFERENCES}

1. Zilberberg MD, Shorr AF. Ventilator-associated pneumonia as a model for approaching cost-effectiveness and infection prevention in the ICU. Curr Opin Infect Dis 2011:(4):385-389.

2. Melsen WG, Rovers MM, Koeman M, Bonten MJ. Estimating the attributable mortality of ventilator-associated pneumonia from randomized prevention studies. Crit Care Med 2011;201(12):27362742 .

3. Ramirez P, Bassi GL, Torres A. Measures to prevent nosocomial infections during mechanical ventilation. Curr Opin Crit Care 2012; 18(1):86-92.

4. Cocanour CS, Peninger M, Domonoske BD, Li T, Wright B, Valdivia A, Luther KM. Decreasing ventilator-associated pneumonia in a trauma ICU. J Trauma 2006;61(1):122-129.

5. Arroliga AC, Pollard CL, Wilde CD, Pellizzari SJ, Chebbo A, Song $\mathrm{J}$, et al. Reduction in the incidence of ventilator-associated pneumonia: a multidisciplinary approach. Respir Care 2011;57(5):688-696. 